\title{
Dynamics of Spindle Assembly of Metal-Cutting Machine
}

\author{
Sh. N. Yaxshiyev, Kh. Kh. Ashurov, A. J. Mamadiyarov
}

\begin{abstract}
The methods of conducting an experiment to study the dynamic characteristics of the elements of the spindle assembly are considered. The results of experimental studies of the dynamics of the spindle assembly of a cutting machine are presented. The method of calculation of dynamic characteristics, and the own frequencies of fluctuation of spindle node of the metal cutting machine "HT-250M» are described. The design of the features of the spindle unit was carried out in the software «APM WINMACHINE».
\end{abstract}

Keywords: spectrum, oscillations, machine tools, dynamics, rigidity, vibrations, diagnostics, metal-cutting machines, spindle, frequency, mathematical modelling.

\section{INTRODUCTION}

Obtaining the surfaces of products of the required quality during machining depends, as a rule, on ensuring the optimal combination of the dynamic state of the machine with technological factors. The instability of the cutting process, which is often manifested during processing, leads to a deterioration in the quality of processing due to the excitation of unacceptably high vibrations in the cutting zone. In order to reduce the probability of forming a deterministic vibration at the frequencies of natural and forced oscillations, the restriction of cutting modes is usually used, which undesirably affects the performance of the process. Improving the dynamic performance of the machine often provides a shift in the permissible limit of modes in the direction of their increase. Hence the conclusion about the potential to improve the quality of processing by improving the dynamic state of the machine. In works $[1,2]$ theoretical study and modeling of dynamic characteristics of spindle units of machines is carried out.

\section{PROPOSED METHODOLOGY}

Spindle unit - one of the most important elements of the dynamic system of the machine, because through the vibrations of the front end of the spindle is affected by the quality and accuracy of processing, durability of the cutting tool, etc. On this basis, the determination of the natural oscillation frequencies spindle unit is necessary at the stage of design and technological design $[1,2,3]$.

Revised Manuscript Received on February 05, 2020.

* Correspondence Author

Sh. N. Yaxshiyev*, Assistant teachers of Navoi state mining institute, Navoi, Uzbekistan

Kh. Kh. Ashurov, Assistant teachers of Navoi state mining institute, Navoi, Uzbekistan

A. J. Mamadiyarov, Assistant teachers of Navoi state mining institute, Navoi, Uzbekistan smoussa@cud.ac.ae

(C) The Authors. Published by Blue Eyes Intelligence Engineering and Sciences Publication (BEIESP). This is an open access article under the CC BY-NC-ND license (http://creativecommons.org/licenses/by-nc-nd/4.0/)
Taking into complexity of the dynamic system of the spindle unit of the NT-250M machine tool and the number of factors that directly affect the formation of the frequency oscillation of the unit, it is necessary to conduct a comprehensive study and determine the most significant elements of the system that must be considered in the modeling process. In order to confirm the theoretical provisions, an experimental study of the spindle Assembly of the machine NT-250M, a simplified image of which is shown in Fig. 1.

To determine the technical condition of the Spindle unit of the machine NT-250M using vibration measurements, a method of collecting and processing information, as well as research to identify operational factors affecting the parameters of the vibration signal.

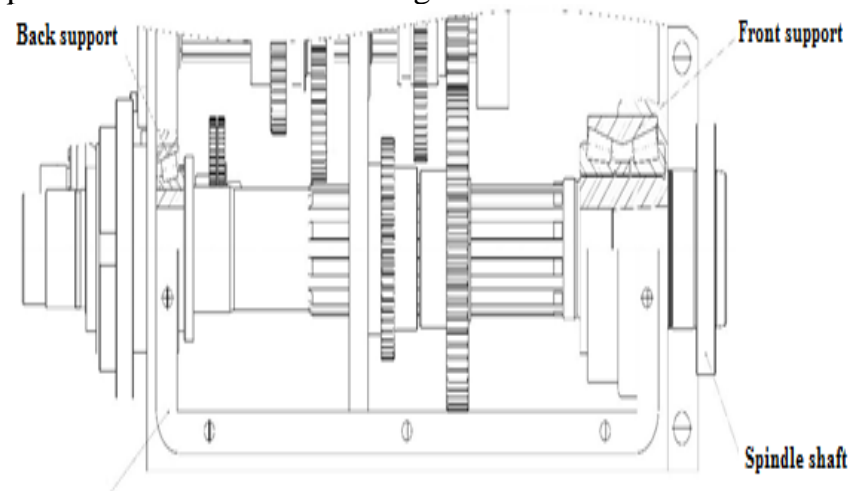

Body

Fig. 1. Simplified image of the spindle Assembly NT250M.

During the experiment, the following characteristics of the spindle unit of vibration displacement, vibration speed and vibration acceleration, as well as the vibration spectra of the elements of the spindle unit NT-250M, were measured and recorded under different processing modes.

The objectives of the research included:

- establishing the dependence of the vibration signal of the spindle Assembly on the operating conditions;

- establishment of criteria of technical condition of the spindle unit of the machine NT-250M;

- establishment of a regime of mandatory control in the diagnosis.

Based on the need to obtain a sufficient amount of data on the technical condition of the spindle unit of the machine NT-250M, the information requirements: continuity, completeness, reliability, homogeneity.

Data on changes in the technical condition of the spindle unit operating under the same conditions, taking into account the ergodic property of random processes in the processing of the material, were recorded on the device "VIBXPERT II" allowing for frequency analysis of the vibration signal. 


\section{Dynamics of Spindle Assembly of Metal-Cutting Machine}

Vibration measurements were made according to the block diagram (Fig. 2), which includes a sensor (piezoaccelerometer), analyzing device (device "VIBXPERT II"), a computer with a software package for vibration monitoring.

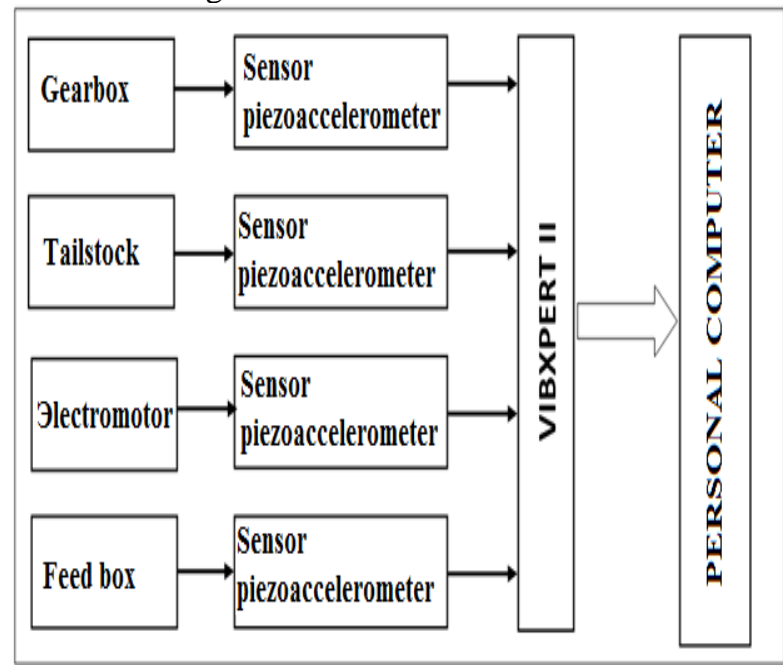

Fig. 2. Block diagram of equipment for vibration analysis machine NT-250M.

A piezoaccelerometer sensor with a wide frequency range was used to convert mechanical vibrations into digital form. Piezoaccelerometers have the smallest measurement error and have a fairly wide frequency range from $5 \mathrm{~Hz}$ to 25 thousand Hz. The mass of the sensor is small in relation to the mass of the studied objects and therefore does not affect the vibrational characteristics.

\section{RESULT ANALYSIS}

In the process of measuring vibration displacements, vibration velocities and vibration accelerations, the piezoelectric sensor was installed with the help of a special magnet on the spindle head body in the immediate vicinity of the supports in the vertical and horizontal direction. The scheme of the experiment is shown in Fig. 3.

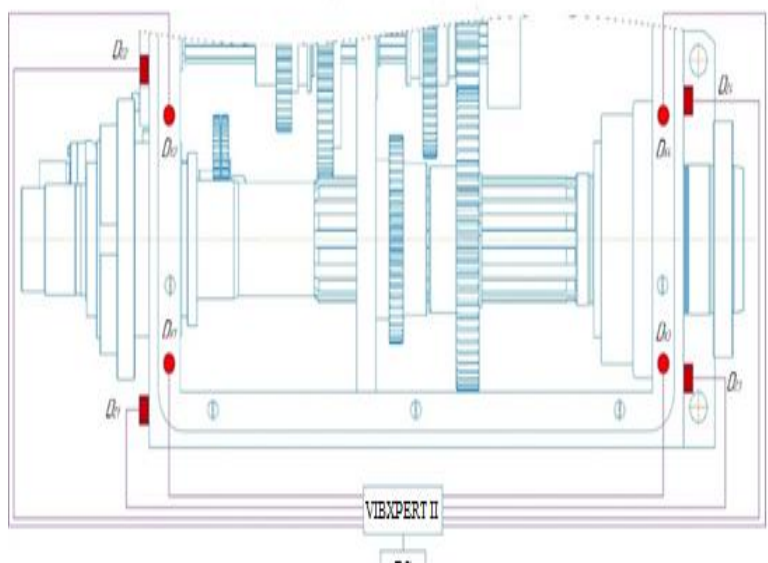

$$
\text { PC }
$$

Fig. 3. Scheme of the experiment.

ED-electric motor; $D_{\mathrm{x} 1}, \mathrm{D}_{\mathrm{x} 2}, \mathrm{D}_{\mathrm{z} 1}, \mathrm{D}_{\mathrm{z} 2^{-}}$piezoelectric accelerometer; VIBXPERT II- portable signal analyzer-data collector VIBXPERT II; PC-personal computer for processing and analysis of measurement results.

In table-I the characteristic frequencies of the bearing units are given, taking into account the kinematic ratios of the bearing elements.
Table-I: Calculation of characteristic frequencies of NT-250M bearings

\begin{tabular}{|c|c|c|c|c|c|c|c|c|c|c|c|c|}
\hline № & $\mathrm{m}, \mathrm{kg}$ & $\begin{array}{l}\mathbb{1}, \\
\mathrm{pc}\end{array}$ & $d, \mathrm{~mm}$ & $\begin{array}{c}\mathrm{D} \\
\mathrm{mm}\end{array}$ & $\begin{array}{c}\mathrm{B} \\
\mathrm{mm}\end{array}$ & pc & $\begin{array}{l}\mathrm{D}_{\mathrm{mw}} \\
\mathrm{mm}\end{array}$ & $\begin{array}{l}\mathrm{F}_{002} \\
\mathrm{~Hz}\end{array}$ & $\begin{array}{l}f_{\mathbb{Z W}} \\
\mathrm{Hz}\end{array}$ & $\mathrm{f}_{\mathrm{N}} \mathrm{Hz}$ & $\mathrm{f}_{8} \mathrm{~Hz}$ & $\mathrm{f}_{\mathrm{B}: \mathrm{Hz}}$ \\
\hline $2.697920 \mathrm{~J} 2$ & 5,49 & 1 & 98,425 & 152,4 & 92 & 26 & 18,5 & 40 & 16,8 & 6,8 & 64 & 76 \\
\hline $4.7716 \mathrm{J1}$ & 3,11 & 1 & 80 & 140 & 77,07 & 14 & 15 & 40 & 30,8 & 10,4 & 415,2 & 144,8 \\
\hline 7308 & 0,703 & 2 & 40 & 90 & 23 & 12 & 13,1 & 33 & 22,8 & 7,9 & 301,8 & 94,2 \\
\hline 36208 & 0,36 & 1 & 40 & 80 & 18 & 9 & 12,7 & 33 & 15,5 & 5,9 & 242,9 & 54,2 \\
\hline 36209 & 0,41 & 2 & 45 & 85 & 19 & 9 & 12,7 & 33 & 16,2 & 6,3 & 239,6 & 7,4 \\
\hline 112 & 0,39 & 1 & 60 & 95 & 18 & 12 & 11,11 & 33 & 15,6 & 5,9 & 323,7 & 72,3 \\
\hline \multicolumn{13}{|c|}{ 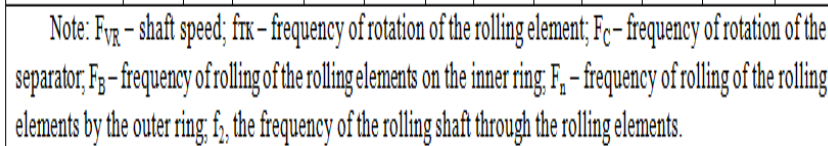 } \\
\hline
\end{tabular}

The results of the measurements were processed using the microprocessor of the device "VIBXPERT II". Each experiment was carried out by 10 linear averaging, which guarantees the reliability of the measurement. In each measurement, the characteristics of the overall level of vibration displacement, vibration velocity, peak-type vibration acceleration and standard deviation, as well as the averaged spectrum of these characteristics were determined. Based on the results of the measurements, the vibration values at the selected frequency were tracked along the available spectrum using the analyzer "VIBXPERT II" on a personal computer, after data transfer from the collector.

According to the results of the experiment, a satisfactory convergence of the eigenfrequency spectra of the system with the data obtained by the analytical data is observed. For fig. 4 shows the dependence of the vibration intensity of the bearing supports of the Spindle unit on the operating parameters of the machine.

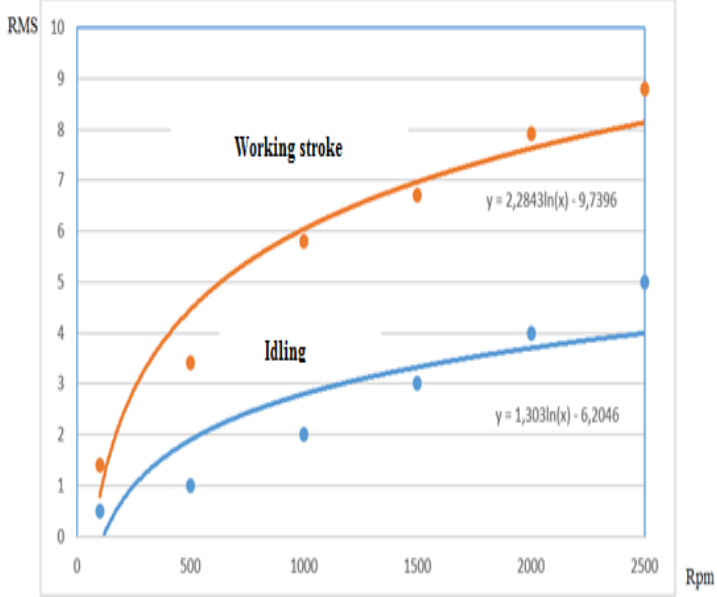

Fig. 4. The dependence of the intensity of the vibration of spindle assembly of the frequency of rotation.

From the experimental dependences, it can be seen that the vibration intensity directly depends on the spindle speed and cutting modes.

Summary. As a result of the analysis, the level of vibration of the machine and its individual units uniquely determines its technical condition.

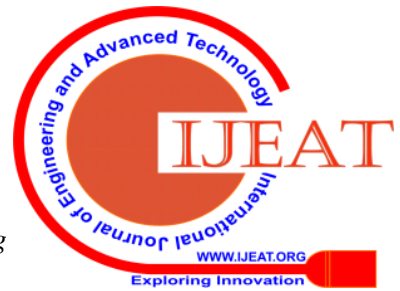


Analysis of experimental data shows that the vibration state of the machine NT-250M significantly depends on the operating parameters of cutting (axial and radial forces, speed, depth of cut and hardness of the processed material). With increasing axial and radial loads, the intensity of vibrations decreases, while with increasing speed and depth of cut it increases;

- with increasing spindle speed PS increases the proportion of high-frequency components in the total dispersion of dynamic loads, while there is a significant increase in the overall level of vibration of the machine;

- spectral analysis shows that the main role in identifying the causes of increased vibration of bearing supports of metal-cutting machines, plays the envelope spectrum;

-on the basis of kinematic and dynamic analysis of the spindle unit, the characteristic vibration frequencies corresponding to certain types of bearing defects spindle unit machine NT-250M.

Dynamic calculation of the spindle assembly of a metal-cutting machine is necessary to determine the dynamic quality indicators used to predict the durability and evaluation of the technical condition of this unit.

For the analysis of dynamics of fluctuations we will use the technique stated In V. A. Kudinov's work [1, 2, 3]. The main assumptions taken when drawing up the design scheme of bearing supports are as follows:

- the reference nodes are considered as a linear dynamic system with distributed and concentrated parameters;

- the shaft and its body (in the case of a cylindrical body) are represented by an elastic beam of step-variable cross-section;

- spindle assemblies have radial, axial and angular stiffness with linear stiffness and damping characteristics;

- the elastic-inertial and damping properties of the shaft and its supports do not change in the angle of rotation of the shaft, i.e. are isotropic.

The input influences on the elastic system of the support unit are the force action (torque) from the electric motor $\mathrm{P}(\mathrm{t})$ and the variable load from the cutter of the holder on which the cutter $\delta(t)$ is located (Fig. 5).

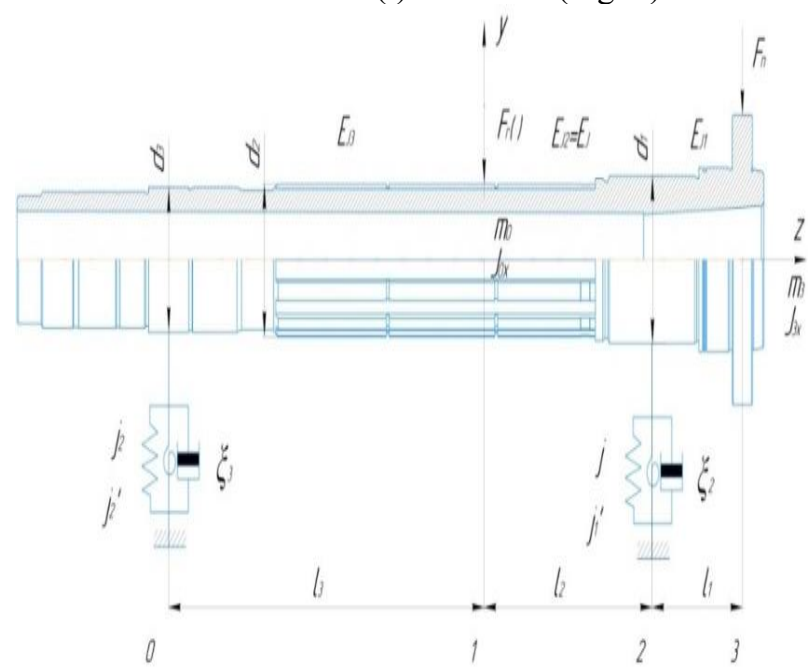

a)

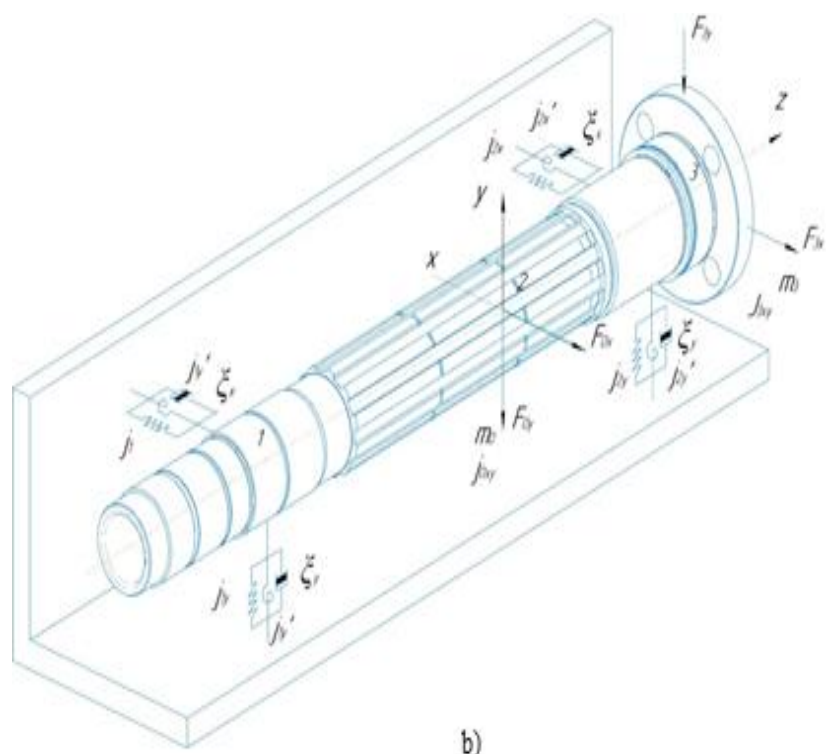

Figure 5. Schematic representation of the elastic-damper elements of the spindle assembly.

Among the numerical methods for calculating the static and dynamic characteristics of spindle units of mechanisms represented as linear elastic systems, the method of initial parameters (in the matrix formulation - the method of transition matrices) has become widespread.

Spindle Assembly (Fig. 5) is considered as a stepped beam of length $l$ on elastic supports with viscous damping proportional to the speed of oscillations. The beam is divided into 3 sections, delimited by a change in diameter (geometric moment of inertia), support, concentrated mass and external concentrated load. Each i- th section has constant (within the section) distributed mass $m_{i}$ and bending stiffness $\mathrm{EJ}_{\mathrm{i}}$.

Mounted on the spindle parts (gearbox, and cutter) are presented in the form of concentrated loads located at both ends of the spindle and having a mass $\mu_{i}$ and moment of inertia $\mathrm{J}_{\mathrm{ix}}$. The calculation of the dynamic characteristics of the spindle unit is reduced to determining the amplitudes of steady-state oscillations of the spindle in the cross-section of the supports from the influence of harmonic force disturbances from the cutting process and the motor drive.

The linear formulation of the problem allows us to use the superposition principle and consistently determine the characteristics of the node under all the above influences.

On the spindle there are concentrated loads-the disturbing force $F_{1}(t)$ and the moment $M_{1}$ acting from the cutter side, as well as the force $F_{2}(t)$ and the moment $M_{2}$ acting from the drive side.

When composing the equation of oscillations of the support nodes in the matrix form of the record we have: 


\section{Dynamics of Spindle Assembly of Metal-Cutting Machine}

$$
[M] \cdot \Delta^{\prime \prime}+[B] \cdot \Delta^{\prime}+[K] \cdot \Delta=F(t),
$$

where the vector of nodal displacements (3n); F(t) - the vector of nodal dynamic loads (3n); [M], [B], [K] - the matrix of mass, damping and stiffness (3nx3n), $n$ - the number of nodes in the design scheme (Fig. 5).

If the perturbation has a steady harmonic character:

$$
F(t)=F_{0} \cdot e^{i \omega t}
$$

the solution of system (3) by the method of complex amplitudes should be sought in the form [2]:

$$
\Delta(t)=\Delta_{0} \cdot e^{i \omega t}
$$

where $\mathrm{F}_{0}$ and $\Delta_{0}$ - complex amplitude values of vectors of nodal loads and displacements; - frequency of perturbation; i-imaginary unit. Substituting (2) and (3) in (1) we obtain

$$
\left([K]-\omega^{2} \cdot[M]+i \cdot \omega \cdot[B]\right) \cdot \Delta_{0}=F_{0} .
$$

Where are the complex amplitudes of oscillations in the supports:

$$
\Delta_{0}=\left([K]-\omega^{2} \cdot[M]+i \cdot \omega \cdot[B]\right)^{-1} \cdot F_{0} .
$$

Assuming that the damping forces in the supports are proportional to the elastic forces, $\omega \cdot[B]=\eta \cdot[K]$, write as:

$$
\left[V^{T}\right]^{-1} \cdot\left([V]^{T} \cdot[K] \cdot[V]-\omega^{2} \cdot[V]^{T} \cdot[M] \cdot[V]+i \cdot \eta \cdot[V]^{T} \cdot[K] \cdot[V]\right) \cdot[V]^{-1} \cdot \Delta_{0}=F_{0} .
$$

Where the vector of complex amplitudes $\Delta_{0}$ is expressed as a matrix of eigenforms [V] and eigenfrequencies $\omega_{j}(\mathrm{j}$ is the eigenfrequency number):

$$
\Delta_{0}[V] \cdot \operatorname{diag}\left[\frac{1}{(1+i \eta) \cdot \omega_{j}^{2}-\omega^{2}}\right] \cdot[V]^{T} \cdot F_{0}=[W] \cdot F_{0} \bar{\Delta}_{0}
$$

given that the modal matrix $[\mathrm{V}]$ has orthogonality properties [2,3,4]:

$$
[V]^{T} \cdot[M] \cdot[V]=[E]_{\text {(Identity matrix), }}[V]^{T} \cdot[K] \cdot[V]=\operatorname{diag}\left[\omega_{j}^{2}\right] \text {. }
$$

Thus, the solution (6) is based on representation of amplitudes of forced oscillations in the form of expansion in a series on forms of natural oscillations that gives essential gain in speed of calculations in comparison with the formula (5). The gain is even more increased if only the lowest forms of oscillations of the support node (3-5 forms) are taken into account. In the case of a relatively short rod elements to account for the influence of transverse forces on distortion of cross sections it is necessary to use modified expressions for the matrices of mass $[\mathrm{M}]^{\mathrm{e}}$ and stiffness $[\mathrm{K}]^{\mathrm{e}}$ elements given in [2, 3].

When composing the equations of oscillations of the elastic system of the support node, we write the following matrices.

The matrix of the inertial characteristics of $\mathrm{M}_{\mathrm{j}}$ in the j-th point:

$$
M_{j}=\left[\begin{array}{llllll}
m_{X} & 0 & 0 & 0 & S_{Z} & -S_{I} \\
0 & m_{I} & 0 & -S_{Z} & 0 & S_{X} \\
0 & 0 & m_{Z} & S_{I} & -S_{X} & 0 \\
0 & -S_{Z} & S_{I} & J_{X} & -\psi_{X I} & -\psi_{X Z} \\
S_{Z} & 0 & -S_{X} & -\psi_{X I} & J_{I} & -\psi_{I Z} \\
-S_{I} & S_{X} & 0 & -\psi_{X Z} & \psi_{I Z} & J_{Z}
\end{array}\right],(7)
$$

specified in the places where the spindle Assembly is attached to the body with the help of bolted connections.

\begin{tabular}{|c|c|c|c|c|c|}
\hline Element & Material & $\begin{array}{l}\text { Density } \\
\text { Kgom }\end{array}$ & $\begin{array}{c}\text { Yulug modulde, } \\
\text { upg }\end{array}$ & Poisson ratio & $\begin{array}{c}\text { Yieds strength, } \\
\text { [Mppa] }\end{array}$ \\
\hline $\begin{array}{l}\text { Spindle of the } \\
\text { machine } \\
\text { HIIS5O-M }\end{array}$ & $\begin{array}{c}\text { CTanb 45 [OCT } \\
1050.2013\end{array}$ & 7810 & 200 & 0,3 & 560 \\
\hline
\end{tabular}
Since the spindle Assembly has two exact diameters for centering the Assembly in the housing, zero displacements along the $\mathrm{x}$ and $\mathrm{Z}$ axes are specified on the specified surfaces, while the $\mathrm{y}$-axis displacements are not limited. Physical and mechanical properties of spindle assembly are given in table-II.

Table-II: Physical and mechanical properties of the elements included in the spindle assembly

Simulation result. In table 3 the first ten natural oscillation frequencies of the spindle unit obtained as a result of modal analysis of the developed mathematical model are given.

where m-masses; J-moments of inertia; $\mathrm{S}$ and $\psi$. respectively static and centrifugal moments of inertia. The body in which the spindle Assembly is installed is conventionally accepted as an absolutely rigid body, therefore, zero displacements along all three axes are 
Table-III: Results of calculation of natural oscillation frequency of spindle unit

\begin{tabular}{|c|c|c|c|c|c|c|c|c|c|c|}
\hline No & 1 & 2 & 3 & 4 & 5 & 6 & 7 & 8 & 9 & 10 \\
\hline $\begin{array}{c}\text { Frequency, } \\
\text { Hz }\end{array}$ & 1327 & 1327.6 & 2039 & 2039.8 & 2862 & 3090 & 3091 & 3502 & 3503 & 4290 \\
\hline
\end{tabular}

As a result of the experiments, the dependences of the vibration amplitude in the bearing supports of the unit at different spindle speeds were established.

Given that the spindle speed range is in the range from 40 to $2500 \mathrm{rpm}$, the maximum frequency of excitation of oscillations, calculated for spindle assembly, will reach $42 \mathrm{~Hz}$, and the minimum $-0.67 \mathrm{~Hz}$. Thus, in this range does not fall any frequency of natural oscillation fluctuations spindle assembly will not affect the accuracy and quality of processing.

\section{1st form of natural oscillations 2nd form of natural oscillations}
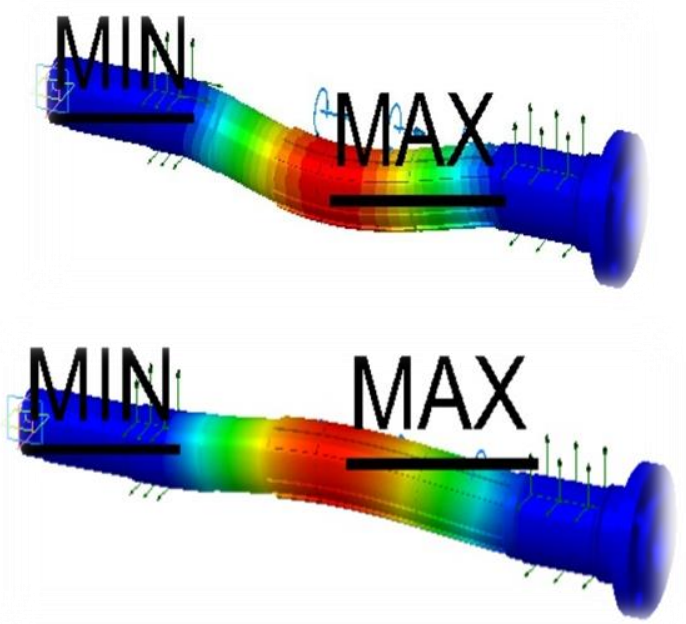

Fig. 6. Forms of natural oscillations of the spindle assembly.

\section{CONCLUSION}

As a result of the analysis of the influence of the parameters of the constituent elements of the spindle Assembly of the lathe NT-250 a mathematical model of the spindle Assembly was developed.

The spindle speed varied in the range from 40 to 2500 revolutions, which correspond to the conditions of real operation of the machine. According to the obtained data, the frequency of oscillations in the supports varied within 12 ... $25 \mathrm{~Hz}$. In turn, the amplitude of the oscillations did not exceed $0.01 \mathrm{~mm}$.

\section{REFERENCES}

1. Automated calculation of dynamic characteristics of technological systems nodes / A. M. Khanov / / Promising technologies and materials: collection of materials of the international scientific and technical conference. - 24 Nov. 2008 / Perm, 2008.Pp. 463-471.

2. Push A.V., Zverev I. A. Spindle assemblies: Design and research. //M., "Stankin", -2000 - P. 197.

3. Kudinov V. A. Dynamics of machines. Moscow: Machine building, 1967. 359 pp.
4. Kudinov V. A. Dynamics of machines. Moscow: Machine building, 1967. 359 pp.

5. Push V. E. Metal-cutting machines. - Moscow: Machine building, 1985. 256 pp.

6. Push A.V., Zverev I. A. Spindle assemblies: Design and research. // M., "Stankin", -2000 - P. 197.

7. Zenkevich O. Finite element Method in engineering. - Moscow: Mir, $1985-542 \mathrm{p}$ 